\title{
Führung I: Bedingungsanalysen und die Entwicklung von Fördermodellen
}

\author{
Telse A. Iwers-Stelljes
}

Die Führung von Unternehmen bzw. Unternehmensbereichen wird aktuell als ein zentraler Wirkfaktor in der Arbeitsorganisation diskutiert. Dabei wird nach außen gerichtet angenommen, dass Führung Marktbedingungen ebenso wie Marketingstrategien beeinflusst; nach innen gerichtete Aspekte erfolgreicher Führung zeigen sich in der Mitarbeitermotivation ebenso wie in deren Gesundheitsstand und Einsatzbereitschaft für jeweilige Arbeitsgruppen und -prozesse. Dabei ist erfolgreiche Führung nicht direkt an Führungsstile oder Führungspersönlichkeiten gebunden, sondern an spezifisches Führungsverhalten (vgl. z. B. Bass und Avoli 1994).

Entscheidende Forschungsperspektiven sind in diesem Kontext, erstens die Faktoren gelungenen Führungsverhaltens differenziert zu evaluieren und zu untersuchen, welche Aspekte gelungenen Führungsverhaltens in Zusammenhang mit personenbezogenen Faktoren der Selbstwirksamkeit, der Arbeitsmotivation und Arbeitsleistung sowie dem Gesundheitserleben und auch der Gruppendynamik in Teams stehen.

Eine zweite Forschungsperspektive ergibt sich aus der Frage des Transfers evaluierter Führungsverhaltensvariablen, d. h. aus der Frage, diese in Führungskompetenztrainings zu überführen. Auch hier zeigen sich aktuell verschiedene Zugänge. So kann Führungskompetenz als genereller Verhaltenskanon definiert werden, zielgruppenspezifisch orientiert spezifiziert werden oder auch subjektiviert als Form von Selbstregulation im beruflichen Kontext etabliert werden. Die Transferorientierung kann überdies daran orientiert sein, bisher eher weniger stark vertretene MitarbeiterInnengruppen stärker in unternehmerische Führungspositionen zu bringen, denn mit Führung geht auch immer die Partizipation an unternehmerischen Entscheidungsprozessen einher.

Die Ankündigung eines Themenheftes zum Thema Führung hat dieser Multiperspektivität entsprechend zu einer Vielzahl breit gestreuter Beiträge geführt. Diese veranlassten zur Konzeption von zwei aufeinander folgenden Heften mit verschiedenen Themenschwerpunkten.

\footnotetext{
Online publiziert: 22.11 .2011

(C) VS Verlag für Sozialwissenschaften 2011

Dr. T. A. Iwers-Stelljes ( $\square)$

Universität Hamburg, Von-Melle-Park 8,

Raum 205a, 20146 Hamburg, Deutschland

E-Mail: GDO@iwers-stelljes.de
} 
In dem vorliegenden Heft werden zunächst mit zwei Beiträgen verschiedene mitarbeiterförderliche Aspekte von Führung differenzieret untersucht.

Sebastian Sauer, Karin Andert, Niko Kohls und Günter F. Müller nehmen in ihrem Beitrag die Perspektive von gesundheitsförderlicher Führung ein. Die AutorInnen untersuchen, inwieweit Achtsamkeit, einer der aktuellen Forschungsschwerpunkte in den Gesundheitswissenschaften, auch für den Bereich Organisationsberatung und Mitarbeiterführung von Belang sein könnte. Ihr Beitrag trägt den Titel Mindful Management: Sind achtsame Führungskräfte leistungsfähigere Führungskräfte?

Jenny Rohde, Sylvie Vincent \& Monique Janneck legen eine geschlechtersensitive Interviewstudie mit (potentiellen) Führungskräften aus technisch-naturwissenschaftlichen Branchen mit dem Ziel der Exploration kompetenz- und karriereförderlicher Führungsverhaltensweisen vor. Diese beinhalten sowohl Förderungen, die eine Führungskraft im direkten Kontakt zeigt, als auch Förderungen über die Gestaltung einer kompetenz- und karriereförderlichen Arbeitsaufgabe. Der Titel ihres Beitrages lautet: Fördern und fordern: Wie Führungskräfte die berufliche Kompetenz- und Karriereentwicklung ihrer Mitarbeiter unterstützen können.

Die anschließenden Beiträge beschreiben zwei sehr unterschiedliche Transferorientierungen.

Günter F. Müller, Martin Sauerland \& Bianca Butzmann fokussieren auf selbstregulative Kompetenzen der Person, indem sie ein Konzept der Führung durch Selbstführung vorlegen und dieses empirisch evaluieren. Ihre Studie zeigt, dass insbesondere die Faktoren ,Coaching' und ,kommunikative Unterstützung' sowie ,Förderung von Freiräumen und Eigenverantwortung' von besonderer Relevanz in ihrem Modell sind. Überdies zeigen sich positive Korrelationen von Führung durch Selbstführung und Bewertung von Vorgesetzten einerseits und allgemeiner Arbeitszufriedenheit andererseits.

Telse Iwers-Stelljes stellt abschließend ein Verfahren der Entwicklung von Führungskompetenz, bestehend aus selbstbezogenen Reflexionsanteilen und interaktionsbezogenen Methodenanteilen vor. Dieses Modul dient der intensiven Verschränkung von selbst- und sozialbezogenen mit methodischen Kompetenzdimensionen. Seine besondere Bedeutung erhält eine solche Verschränkung im Hinblick auf die weibliche Adressatengruppe, hier sind es exemplarisch weibliche Verwaltungskräfte im kommunalen Bereich, deren stärkere Orientierung an ,soft skills‘ und weichen Führungsvariablen eine deutliche Methodenorientierung beigefügt wird. Der Beitrag heißt: Führungskompetenz durch die Verschränkung von Selbstreflexion und Methodenkenntnis am Beispiel eines Führungskompetenztrainings für weibliche Verwaltungskräfte im kommunalen Bereich.

Im zweiten Heft zum Thema Führung wird zunächst ein Beitrag von Ivette Voelschow zur Entwicklung eines kollegialen Coachings für Führungskräfte aus Polizei und Justiz präsentiert, der sich an der Schnittstelle zwischen Bedarfsevaluation und Praxistransfer befindet.

Im Anschluss wird verstärkt auf genderspezifische Fragestellungen von Führung fokussiert. So wird von Judith Oerding eine Studie zu den Anliegen weiblicher Führungsnachwuchskräfte in Mentoringprozessen vorgelegt. Von Daniela Rastetter \& Doris Cornils wird Networking als aufstiegsförderliche Strategie für Frauen in Führung dargestellt. Sylvie Vincent legt eine Untersuchung zu gesundheits- und entwicklungsförderlichem Führungsverhalten unter genderspezifischer Perspektive vor und Jenny Rohde, Sylvie 
Vincent und Monique Janneck liefern einen Überblick über aktuelle Literatur zu genderspezifischen Führungsaspekten.

\section{Literatur}

Bass, B. M., \& Avolio, B. J. (1994). Improving organizational effectiveness through transformational leadership. California: Sage 